\title{
The inversion of the Lorentz integral transform with controlled resolution
}

\author{
Winfried Leidemann ${ }^{1,2 \star}$, Victor D. Efros ${ }^{3,4}$ and Veronica Yu. Shalamova ${ }^{3}$
}

1 Dipartimento di Fisica, Università di Trento, 38123 Trento, Italy

2 INFN-TIFPA Trento Institute of Fundamental Physics and Applications, 38123 Trento, Italy

3 National Research Centre "Kurchatov Institute", 123182 Moscow, Russia

4 National Research Nuclear University MEPhI (Moscow Engineering Physics Institute), Russia

* winfried.leidemann@unitn.it

October 15, 2019

Proceedings for the 24th edition of European Few Body Conference, Surrey, UK, 2-4 September 2019

\begin{abstract}
The Lorentz integral transform (LIT) method is briefly presented. The ill-posedness of the inversion is discussed and it is pointed out that the LIT is an integral transform with a controlled resolution. As application the dipole response of a three-particle system bound in a hypercentral potential is considered. The inversion is performed in two ways, both employ expansions of the response function and of the LIT on a basis set and a transformed basis set, respectively. A previous study of the process under consideration seemed to point to serious problems in the inversion process. In the present work, however, it is shown that the LIT approach can be carried out in a reliable way.
\end{abstract}

\section{Introduction}

Integral transforms are a powerful tool in physics. In general they have the following form

$$
\Phi_{K}(\sigma)=\int d \omega K(\omega, \sigma) R(\omega)
$$

where $R(\omega)$ is a response function containing information about a physical system, $K(\omega, \sigma)$ is a kernel and $\Phi_{K}(\sigma)$ is the integral transform resulting from kernel $K$. Integral transforms become particularly interesting for cases, where it is difficult or even impossible to determine the response $R$, but where the calculation of the transform $\Phi_{K}$ is much less problematic. An example for such a transform is the LIT, the Lorentz integral transform, which will be defined in the following section. It has been introduced more than twenty years ago [1] and since then quite a number of applications have been published (see e.g. [2]).

The strength of the LIT technique lies in the fact that a continuum state problem is reduced to a bound-state like one. There is, however, an obstacle to overcome. In fact one is confronted with an ill-posed problem, namely the inversion of the transform. Applying a regularization procedure the obstacle can be overcome. Still in specific cases problems can arise. Such a case is given by the dipole response of a three-particle system bound in a hypercentral potential, which has been discussed in [3]. The authors of this article fail in inverting the LIT correctly. 
In the present work we reconsider exactly the same problem. As it will be shown we perform the inversion in two somewhat different ways. Both ways lead to reliable results, but one of them is actually better suited in order to invert the LIT with less numerical effort and in addition in a more controllable manner.

\section{Lorentz integral transform}

The LIT $L(\sigma)$ is defined as follows

$$
L(\sigma)=\int d \omega \frac{R(\omega)}{\left(\omega-\sigma_{R}\right)^{2}+\sigma_{I}^{2}},
$$

where $\sigma$ is a complex variable: $\sigma=\sigma_{R}+i \sigma_{I}$.

Here we restrict our discussion to such inclusive response functions that are defined by the relation

$$
R(\omega)=\sum_{n}|\langle n|\hat{O}| 0\rangle|^{2} \delta\left(E_{n}-E_{0}-\omega\right)+\int d f|\langle f|\hat{O}| 0\rangle|^{2} \delta\left(E_{f}-E_{0}-\omega\right),
$$

where $|0\rangle,|n\rangle$ and $|f\rangle$ are ground, bound and continuum states of a particle system that undergoes a reaction induced by a transition operator $\hat{O}$, while $E_{0}, E_{n}$ and $E_{f}$ are the corresponding eigenenergies. The LIT of the response function above is given by

$$
L(\sigma)=\langle\tilde{\Psi} \mid \tilde{\Psi}\rangle,
$$

where $\tilde{\Psi}$ satisfies the following equation

$$
\left(H-E_{0}-\sigma\right) \tilde{\Psi}=\hat{O}|0\rangle
$$

with $H$ being the Hamilton operator of the particle system. Note that $\tilde{\Psi}$ can be determined with bound-state methods although the response function includes transition to final states in the continuum. In fact the LIT method is particularly advantageous for the calculation of response functions for reactions into the many-body continuum.

There is an alternative formulation of the LIT,

$$
L(\sigma)=-\frac{1}{\sigma_{I}} \operatorname{Im}\left(\left\langle 0\left|\hat{O}^{\dagger} \frac{1}{\sigma_{R}+i \sigma_{I}+E_{0}-H} \hat{O}\right| 0\right\rangle\right),
$$

which is useful in case of an expansion of $\tilde{\Psi}$ on a complete many-body basis set with a subsequent use of the Lanczos method [4].

In order to obtain the response function $R(\omega)$ one has to invert the LIT. In case that transitions to bound states exist one should first determine the corresponding response function $R\left(E_{n}-E_{0}\right)$ for the various bound states $E_{n}$ and subtract their contributions to the LIT. Note that these determinations are easily achieved by choosing a very small value for $\sigma_{I}$ and setting $\sigma_{R}=E_{n}-E_{0}$. After having determined in this way the LIT of the response function beyond the break-up threshold of the particle system one can proceed to its inversion. Different LIT inversion methods have been used [5]. A very robust technique that has been applied in most of the LIT applications is based on a formal expansion of $R(\omega)$ on a specific basis set,

$$
R(E)=\sum_{n=1}^{N} c_{n} f_{\mathrm{thr}}(E) \exp (-\alpha E / n),
$$


where $E=\omega-\omega_{\text {thr }}$ with $\omega_{\text {thr }}$ being the break-up threshold of the particle system and where the function $f_{\text {thr }}(E)$ describes the threshold behaviour of the response function. The LIT of the formal response of Eq. (7) is then given by

$$
L(\sigma)=\sum_{n=1}^{N} c_{n} \int_{\omega_{\mathrm{thr}}}^{\infty} d \omega \frac{f_{\mathrm{thr}}(E) \exp (-\alpha E / n)}{\left(\omega-\sigma_{R}\right)^{2}+\sigma_{I}^{2}} .
$$

The coefficients $c_{n}$ are determined via a fit to the calculated LIT. To this end the LIT should be calculated for a fixed $\sigma_{I}$ and a sufficiently large number of $\sigma_{R}$ values. Increasing $N$, the number of basis functions, one should find a reasonable convergence for the resulting response functions. Note that too large values of $N$ can lead to instabilities due to the illposedness of the inversion and that the above mentioned regularization consists just in such a limitation of $N$.

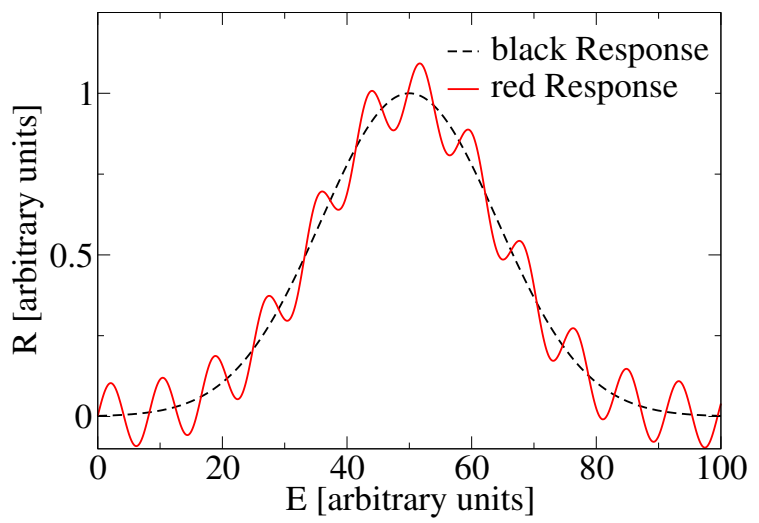

Figure 1: Two artificial response functions: a "black" response $R_{\text {black }}(E)$ (black dashed) and a "red" response $R_{\text {red }}(E)$ (red solid).

\section{LIT: an integral transform with controlled resolution}

Different from many other integral transforms one can assign a well-defined resolution to the LIT. In fact, the resolution is determined by the size of $\sigma_{I}$. As we will see in section 4 this helps in not running into the problem of ill-posedness. Ill-posedness means here that two rather different response functions can lead to two rather similar integral transforms. In order to illustrate this fact we show in Fig. 1 such an example with a "black" $\left(R_{\text {black }}(E)\right)$ and a "red" response $\left(R_{\text {red }}(E)\right)$. The black response $R_{\text {black }}$ is rather smooth, whereas the red response $R_{\text {red }}$ exhibits oscillations about the black response. In the left panel of Fig. 2 the corresponding LITs, $L_{\text {black }}(\sigma)$ originating from $R_{\text {black }}$ and $L_{\text {red }}(\sigma)$ originating from $R_{\text {red }}$, are shown for $\sigma_{I}=10$ units. Though the two responses are rather different there is no visible difference for the two LITs. It shows that it would be difficult to make inversions of these two LITs such that both original responses are recovered. In fact it would only be possible if both LITs are known with an extremely high precision. In general such a precision cannot be obtained in realistic calculations. On the other hand one can try to calculate the LIT with a higher resolution and thus with a smaller $\sigma_{I}$. However, one has to be aware that a higher resolution might require a higher precision for the calculation of the LIT. Therefore, in general, in a given calculation there will be a natural limit for the minimal size of $\sigma_{I}$.

Since for the example shown in Fig. 1 we have analytic expressions for both responses there is no problem to calculate the LITs with a smaller $\sigma_{I}$ than 10 units. In the right panel of 

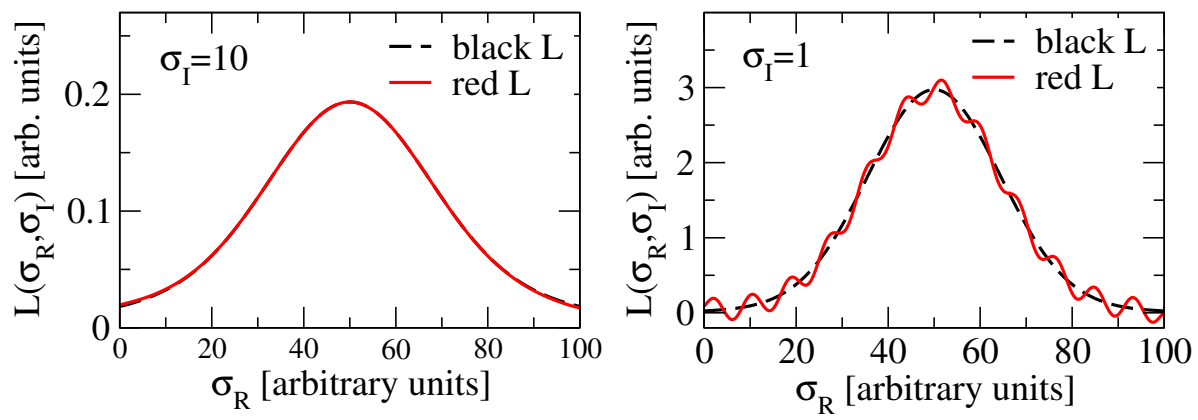

Figure 2: The LITs of black (black dashed) and red responses (red solid). Left panel: $\sigma_{I}=10$ units. Right panel: $\sigma_{I}=1$ unit.

Fig. 2 results with $\sigma_{I}=1$ unit are shown. One readily sees that in this case the resolution is high enough to show also for $L_{\text {red }}$ the presence of oscillations in $R_{\text {red }}$.

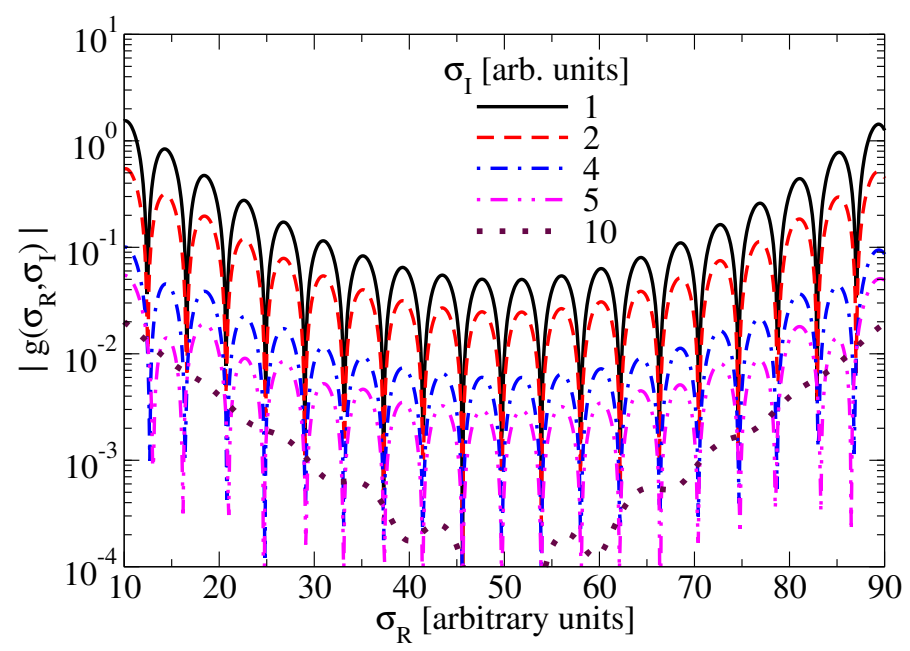

Figure 3: The absolute value of $g\left(\sigma_{R}, \sigma_{I}\right)$ for the $\sigma$ equal to $1,2,4,5$, and 10 units .

In order to better illustrate the differences between the black and red LITs we define the following function

$$
g\left(\sigma_{R}, \sigma_{I}\right)=\frac{L_{\text {red }}\left(\sigma_{R}, \sigma_{I}\right)-L_{\text {black }}\left(\sigma_{R}, \sigma_{I}\right)}{L_{\text {black }}\left(\sigma_{R}, \sigma_{I}\right)} .
$$

In Fig. 3 we show the absolute value of $\left.g\left(\sigma_{R}, \sigma_{I}\right)\right)$ for various $\sigma_{I}$. For $\sigma_{I}=10$ units it is again evident that differences between black and red LITs are very small. In addition one sees that the oscillatory behaviour of the red response is not all reflected. As the figure shows one has to reduce $\sigma_{I}$ to about half of size of the "wave length" of the oscillations of the red response, which is a little bit less than 5 units. One also notes that further decreases of $\sigma_{I}$ make the oscillations more and more pronounced. These results nicely demonstrate that the LIT is an integral transform with a controlled resolution.

\section{Dipole response of a three-nucleon system with a hypercentral interaction}

In this section we reconsider the dipole response of a three-particle system calculated in [3]. There it was assumed that two of the three particles are neutrons, whereas the third particle 
is either a proton or an $\alpha$-particle. Here we consider the three-nucleon case, where the third particle is a proton. The transition operator to consider in this case is the dipole operator,

$$
\mathbf{d}=e \sum_{i=1}^{3} \frac{1+\tau_{i, z}}{2} \mathbf{r}_{i},
$$

where $\tau_{i, z}$ and $\mathbf{r}_{i}$ are the third component of the isospin operator and the position of the $i$-th nucleon, respectively. The hypercentral interaction used in [3] reads as follows

$$
V(\rho)=V_{0} \exp \left(-\kappa \rho^{2}\right)
$$

with $V_{0}=-75 \mathrm{MeV}$ and $\kappa=0.16 \mathrm{fm}^{-2}$.

The solution of the Schrödinger equation for the ground state and the by the dipole operator induced final state is relatively simple, since the model can be reduced to an effective one-body problem, where the hypercentral potential is replaced by a central one. Then, in the ground state, one has a nucleon with "orbital momentum" $3 / 2$, while in the final state the nucleon carries "orbital momentum" 5/2. The solutions of Schrödinger and LIT equations (5) were obtained via expansions on harmonic oscillator basis sets. Results have been determined with a very high precision for (i) energy and radius of the ground state, (ii) the LIT and (iii) for the phase shifts of the relevant partial wave of the final continuum state. For further details concerning both the calculation and explicit results we refer to [6].

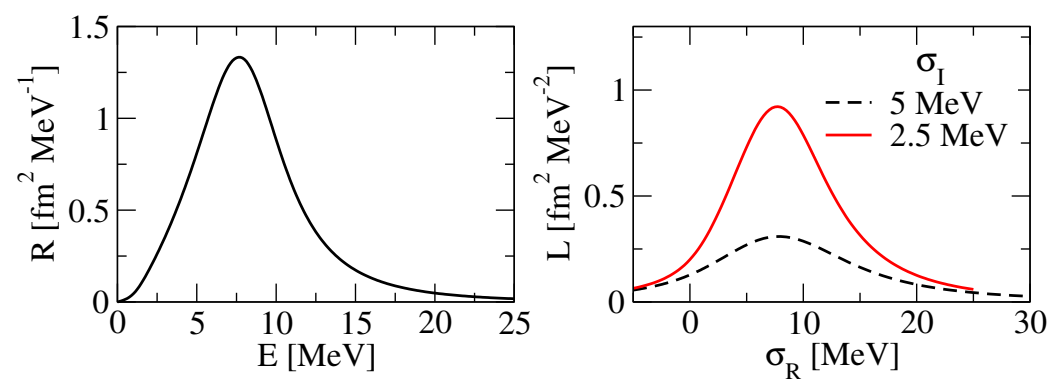

Figure 4: Left panel: the dipole response function R(E); right panel: the LITs of R(E) for $\sigma_{I}=5 \mathrm{MeV}$ (black dashed) and $\sigma_{I}=2.5 \mathrm{MeV}$ (red solid).

In the left and the right panel of Fig. 4 we show the response function and the LIT, respectively. The response exhibits a peak with a maximum at about $8 \mathrm{MeV}$. Low-energy increase and high-energy decrease are very smooth and do not have additional structures. The LITs show a very similar behaviour. The peak height of $L\left(\sigma_{R}, \sigma_{I}=2.5 \mathrm{MeV}\right)$ is almost four times higher than that of $L\left(\sigma_{R}, \sigma_{I}=5 \mathrm{MeV}\right)$, which is easily understood by investigation of Eq. (2).

For the inversion of the calculated LIT we employed two different methods both based on the expansions given in Eqs. (7) and (8). Details are explained in the following.

\subsection{Inversion with quadrupole precision}

The basis of Eq. (7) is used for the inversion and the proper threshold behaviour for the present case is taken: $f_{\text {thr }}(E)=E^{3}$. The inversion has been made with a great numerical precision, in particular a quadrupole precision has been used for the determination of the nonlinear parameter $\alpha$ and the coefficients $c_{n}$ (for details see [6]). The reason for this extremely high precision is due to the relative large number of employed basis functions: $30 \leq N \leq 50$. In this case the higher $c_{n}$ become very large in magnitude and reach values up to about $10^{10}$ and the resulting corresponding contributions strongly cancel each other. As illustrated in [6] the inversion leads to excellent results with exception of the response at very low energies. 
Therefore, in Fig. 5 we only show the response below $6 \mathrm{MeV}$. In the left panel of the figure the inversions for $\sigma_{I}=5 \mathrm{MeV}$ are depicted for various $N$ values. One sees that the convergence of the results is extremely good for $E \geq 3 \mathrm{MeV}$, but that the convergence is different at lower energies. In particular for $E=2 \mathrm{MeV}$ the result is very unsatisfactory. In fact with the highest number of basis functions, $N=50$, the difference to the true response becomes larger which is a sign that one runs into the ill-posedness of the inversion. Of course, in a normal application of the LIT one would not know the true response, but nonetheless one would realize that the $N=50$ inversion had to be excluded and, in addition, an error for the low-energy response had to be assigned. On the other hand one can try to increase the resolution by decreasing $\sigma_{I}$. Such results with $\sigma_{I}=2.5 \mathrm{MeV}$ are shown in the right panel of Fig. 5 and in fact there one sees a great improvement of the results. Now one finds a very good convergence also at low energy and in addition a very good agreement with the true response.

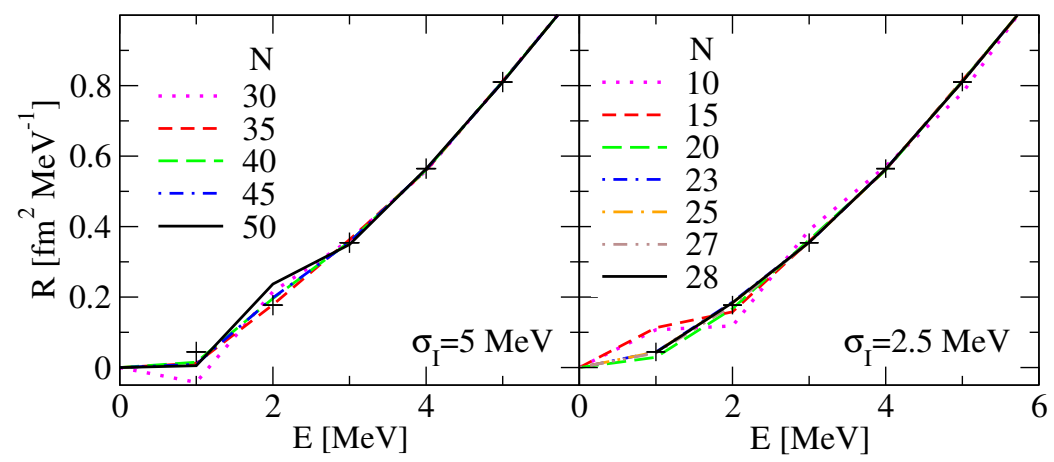

Figure 5: Left panel: Inversions of $L\left(\sigma_{R}, \sigma_{I}=5 \mathrm{MeV}\right)$ with various numbers $N$ of basis functions for the inversion (see Eqs. (7), (8)); right panel: inversions as in left panel but for $L\left(\sigma_{R}, \sigma_{I}=2.5 \mathrm{MeV}\right)$. Plus signs describe exact response.

\subsection{Standard inversion}

For our standard inversion the calculation is made in double precision and the main difference to the inversion method discussed in subsection 4.1 consists in discarding some of the inversion solutions. The criterion for the removal are (i) the response is not positive definite or (ii) the response shows structures not visible in the transform. Concerning criterion (i) one has to take into account that different from our artificial example in Fig. 1 an inclusive response function cannot become negative.

Applying these criteria to the inversions of Fig. 5 one should drop the cases with $N=30$ (criterion (i)) and those with $N=10,15,50$ (criterion (ii)). Different from our standard method the next best inversion solution has not been defined in subsection 4.1. Contrarily, in the standard approach we determine solutions for a large number of values of the nonlinear parameter $\alpha$ and arrange them according to the quality of the fit to the calculated LIT and subsequently apply the two criteria. Thus, the chosen inversion is the best of all solutions that fulfills the criteria (i) and (ii).

In the upper panel of Fig. 6 we show the converged inversion results with a $\sigma_{I}$ of 2.5 and $5 \mathrm{MeV}$. As in Fig. 5 we find an improvement with the higher resolution, but the result for $\sigma_{I}=2.5 \mathrm{MeV}$ is not as precise as with the inversion with quadrupole precision. Even not knowing the true response one may conclude that the inversion of the low-energy response needs a further improvement: a closer inspection of the inversion exhibits a slight oscillation which is not present in the transform. In a real application a quadrupole precision is most probably prohibitive, but a further reduction of $\sigma_{I}$ might be possible. Another possibility is the choice of a different basis set for the inversion. In the present case, where one finds 


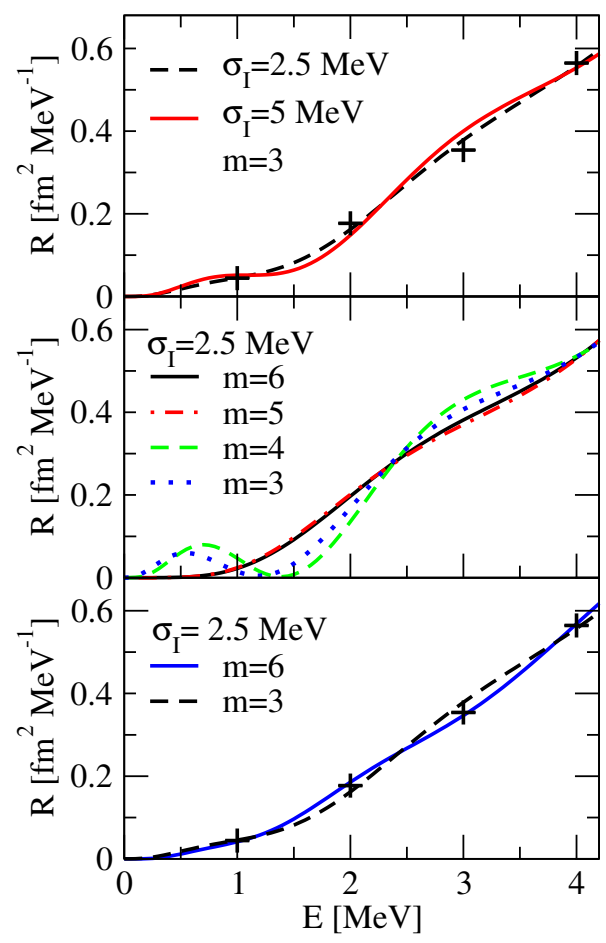

Figure 6: Upper panel: converged inversion results with $\sigma_{I}$ equal to 5 (red solid) and $2.5 \mathrm{MeV}$ (dashed black); middle panel: Inversion results with $N=8$ for basis sets with threshold functions $f_{\text {thr }}=E^{m}$ with different exponents $m$, namely $m=3,4,5,6$ (see Eqs. (7), (8)); lower panel: converged inversion results with $\sigma_{I}=2.5 \mathrm{MeV}$ and $m=3$ (black dashed) and $m=6$ (blue solid). Plus signs describe exact response.

problems in the threshold region one can modify the threshold behaviour of the response given by $f_{\text {thr }}$. Therefore, in the middle panel of Fig. 6, we show inversions with basis sets, where we have set $f_{\text {thr }}=E^{m}$ with $m=3,4,5,6$ and where the number of basis functions $N$ is restricted to 8 . One sees quite a difference for the results with $m=3,4$ on the one hand and those with $m=5,6$ on the other hand: strong oscillations of the response are only present for the former. Thus $f_{\text {thr }}=E^{m}$ with the higher exponents $m$ of 5 and 6 seem to be more suited for the inversion. This is not in contradiction with the true threshold behaviour with $m=3$, since in general it is unknown for which energy interval the true threshold behaviour holds, for example, the interval could be very small. In the lower panel of Fig. 6 we show the converged inversion result with a modified threshold behaviour, $f_{\text {thr }}=E^{6}$, in comparison to that with $f_{\text {thr }}=E^{3}$. In fact, one finds a further improvement of the result, but one can still note that the response exhibits a structure not present in the transform. Thus, for a very precise threshold result a further decrease of $\sigma_{I}$ is necessary. Actually the modified threshold behaviour does not only positively affect the low-energy region but, as illustrated in Table 1 , leads also to an improvement of the inversion in the peak region. This is probably explained by the fact that the better threshold result for the $m=6$ case leaves more space to further improve the fit in other energy regions.

\section{Conclusion}

We have shown that reliable results for inclusive response functions can be obtained with the Lorentz integral transform method. However, since the inversion of the LIT is an ill- 
Table 1: The exact response $R(E)$ and those obtained via inverting the LIT for basis sets with $f_{\text {thr }}=E^{m}$ and $m=3$ and $m=6$.

\begin{tabular}{|c|c|c|c|}
\hline$E[\mathrm{MeV}]$ & exact $\mathrm{R}\left[\mathrm{fm}^{2} \mathrm{MeV}^{-1}\right]$ & $R\left[\mathrm{fm}^{2} \mathrm{MeV}^{-1}\right] m=3$ & $R\left[\mathrm{fm}^{2} \mathrm{MeV}^{-1}\right] m=6$ \\
\hline 7 & 1.284 & 1.296 & 1.289 \\
8 & 1.321 & 1.309 & 1.320 \\
9 & 1.150 & 1.142 & 1.148 \\
\hline
\end{tabular}

posed problem one has to invert the LIT with great care. As an interesting example, where in the past a LIT application has led to problems, we have considered the dipole response function of a three-particle system bound in a hypercentral potential. The inversion has been made with two slightly different procedures, one with a quadrupole precision and the other one via our standard inversion method. Comparing with the exact response, results with a satisfying precision are obtained beyond the threshold region. Only at low energies problems arise, which, as shown however, can be largely resolved by increasing the resolution of the transform. Also a different choice of the basis functions for the inversion helps in this case.

It is important to note that one can realize the existence of inversion problems in the low-energy region without the knowledge of the exact response function. In this context it is important to know that the LIT is a transform with a controlled resolution and in order to have a safe inversion one should not accept inversions which exhibit structures that are below the resolution of the transform. If one wants to investigate whether such structures are real one has to increase the resolution of the LIT.

Funding information Acknowledgement of support is given to RFBR Grant No. 18-0200778 (V.D.E and V.Yu.S.).

\section{References}

[1] V. D. Efros, W. Leidemann and G. Orlandini, Response functions from integral transforms with a Lorentz kernel, Phys. Lett. B 338, 130 (1994), doi:10.1016/03702693(94)91355-2.

[2] V. D. Efros, W. Leidemann, G. Orlandini and N. Barnea, The Lorentz integral transform (LIT) method and its application to perturbation-induced reactions, J. Phys. G: Nucl. Part. Phys. 34, R459 (2007), doi:10.1088/0954-3899/34/12/R02.

[3] Y. Suzuki, W. Horiuchi and D. Baye, Greene's function method for strength function in three-body continuum, Prog. Theor. Phys. 123, 547 (2010), doi:10.1143/PTP.123.547.

[4] M. A. Marchisio, N. Barnea, W. Leidemann and G. Orlandini, Efficient method for Lorentz integral transforms of reaction cross sections, Few-Body Syst. 33, 259 (2003), doi:10.1007/s00601-003-0017-z.

[5] D. Andreasi, W. Leidemann, Ch. Reiss and M. Schwamb, New inversions methods for the Lorentz integral transform, Eur. Phys. J. A. 24, 361 (2005), doi:10.1140/epja/i200510009-3.

[6] V. D. Efros, W. Leidemann and V. Yu. Shalamova, On calculating response functions via their Lorentz integral transforms, Few-Body Phys. 60, 35 (2019), doi:10.1007/s00601019-1497-9. 